Article

\title{
Comparison and Identification of the Aroma-Active Compounds in the Root of Angelica dahurica
}

\author{
Die Hu ${ }^{1,2} \mathbb{D}_{\text {, Junrui Guo }}{ }^{1,2}$, Ting $\mathrm{Li}^{1,2}$, Mu Zhao ${ }^{1,2}$, Tingting Zou ${ }^{1,2, *}$, Huanlu Song ${ }^{1,2}$ and \\ Aygul Alim ${ }^{3}$ \\ 1 Beijing Higher Institution Engineering Research Center of Food Additives and Ingredients, Beijing \\ Technology and Business University (BTBU), Beijing 100048, China; tianshijie2525@163.com (D.H.); \\ gjr990316@163.com (J.G.); liting120902@163.com (T.L.); zhaomu2985@163.com (M.Z.); \\ songhl@th.btbu.edu.cn (H.S.) \\ 2 Beijing Key Laboratory of Flavor Chemistry, Beijing Technology and Business University (BTBU), \\ Beijing 100048, China \\ 3 College of Food Science and Pharmacy, Xinjiang Agriculture University, Xinjiang 830052, China; \\ aygulalim@sina.com \\ * Correspondence: zoutingting@th.btbu.edu.cn
}

Received: 3 November 2019; Accepted: 26 November 2019; Published: 28 November 2019

\begin{abstract}
Solid-phase microextraction (SPME), purge and trap (P\&T), stir bar sportive extraction (SBSE), and dynamic headspace sampling (DHS) were applied to extract, separate and analyze the volatile compounds in the roots of Hangbaizhi, Qibaizhi, and Bobaizhi and the GC-O-MS/MS (AEDA) was utilized for the quantification of key aroma compounds. Totals of 52, 54, and 43 aroma-active compounds extracted from the three samples by the four extraction methods were identified. Among these methods, the SPME effectively extracted the aroma compounds from the A. dahurica. Thus, using the SPME methods for quantitative analysis based on external standards and subsequent dilution analyses, totals of 20, 21, and 17 aroma compounds were detected in the three samples by the sniffing test, and sensory evaluations indicated that the aromas of $A$. dahurica included herb, spice, and woody. Finally, principal component analysis (PCA) showed that the three kinds A. dahurica formed three separate groups, and partial least squares discriminant analysis (PLS-DA) showed that caryophyllene, (-)- $\beta$-elemene, nonanal, and $\beta$-pinene played an important role in the classification of A. dahurica.
\end{abstract}

Keywords: Angelica dahurica root; aroma-active compounds; solid-phase microextraction (SPME); gas chromatography-olfactometry-mass spectrometry (GC-O-MS); aroma extract dilution analysis (AEDA)

\section{Introduction}

Angelica dahurica (Baizhi) is a traditional herb in China, according to the location of origin the A. dahurica has 5 types: Hangbaizhi, Qibaizhi, Chuanbaizhi, Yubaizhi, and Bobaizhi [1]. The Angelica dahurica is a dual-purpose plant with medicinal and edible value. On the one hand, with the increasing health consciousness, A. dahurica will be increasingly popular, as it has anti-inflammatory, antioxidant, antibacterial, and anticancer properties [2-5]. Root of Angelica dahurica was defined in the 2010 edition of the China Pharmacopoeia (2010) [6] as the dry root of Angelica dahurica (Fisch.ex Hoffm.) Benth.et Hook.f. or Angelica dahurica (Fisch.ex Hoffm.) Benth.et Hook.f. var. formosana (Boiss.) Shanet Yuan. On the other hand, its edible parts are used as a spice for flavoring, seasoning, or imparting aroma. In previous work, out of the very few studies on the flavor of the root of A. dahurica, $\mathrm{Li}$, Lu and Feng [7] use gas chromatography-mass spectrometry (GC-MS) to analyze the volatile components, such as 1,8-cineol, limonene and caryophyllene (SPME). Furthermore, the terpenes, 
$\alpha$-limonene, 3-decene, and 1-R- $\alpha$-pinene were identified in the volatile oil of $A$. dahurica [8], and $\mathrm{Li}$ et al. [9] found that dodecanal is the most abundant species in the volatile oil of Chuanbaizhi and Hangbaizhi and found that volatile oils have many different flavors.

However, a comprehensive analysis of the key aroma-active compounds of the root of A. dahurica has not been reported. In further investigations focusing on the flavor, some scholars have studied the aroma components of botanical seasonings [10-13]. Omar et al. [12] used dynamic headspace sampling-gas chromatography-mass spectrometry (DHS-GC-MS) to analyze the monoterpenoids in four aromatic plants [14]. Raffo et al. [13] identified the key aroma-active components in rocket leaves by combining headspace solid-phase microextraction (HS-SPME), stir bar sportive extraction (SBSE) with GC-MS, and gas chromatography-olfactometry (GC-O); they found that the most potent odor-active compounds identified included (Z)-3-hexenal, (E)-3-hexenal, (Z)-1,5-octadien-3-one, and 4-mercaptobutyl [15]. However, the summary on the characteristics of odorents on the different of types A. dahurica, especially, qualification and quantification on the aroma compounds was still fragmentary.

The main objectives of this study were, firstly, to compare the four headspace analysis methods (SPME, P\&T, SBSE, and DHS) to identify the volatile flavor compounds from the three types of $A$. dahurica. Secondly, SPME-AEDA and flavor dilution (FD) factors were employed to identify and quantify ten key aroma-active compounds shared by the three types of A. dahurica. Finally, the $A$. dahurica samples were divided into three groups by principal component analysis (PCA), and partial least squares discriminant analysis (PLS-DA) was used to identify the aroma-active compounds that most strongly influenced the classification.

\section{Results and Discussion}

\subsection{Comparison of Quantity of Aroma Compounds by the Different Extraction Methods}

Three replicate experiments using the same sample were performed on the same day to test the repeatability of each method. The coefficients of variation ( $\mathrm{CV}$ values) of the total ion counts for SPME, P\&T, SBSE, and DHS are $1.40 \%, 5.65 \%, 6.76 \%$, and $6.441 \%$, respectively, indicating that SPME was more repeatable than the other three methods.

The compounds extracted by the four headspace extraction methods and analyzed by GC-O-MS are shown in Table 1 and Figure 1. A total of 52,54, and 43 aroma compounds were identified in the samples from Hangbaizhi, Qibaizhi, and Bobaizhi, respectively. These compounds included terpenes, aromatic species, alcohols, aldehydes, ketones, acids, and esters. Moreover, a total of 20, 21, and 17 aroma-active compounds were detected in the A. dahurica samples from Hangbaizhi, Qibaizhi and Bobaizhi, respectively, by the sniff test. These compounds contributed aroma characteristics of pine, camphor, woody, herb, citrus, spice, cucumber, sour, and so on. We also found that the most abundant type of compounds in all three types of A. dahurica were terpenes, with 28, 30, and 24 derivatives detected in Hangbaizhi, Qibaizhi, and Bobaizhi, respectively, followed by aldehydes with 9, 8, and 7 derivatives identified in Hangbaizhi, Qibaizhi and Bobaizhi, respectively. Among these, 1,8-cineol, limonene and caryophyllene have been detected previously from the root of A. dahurica [7], to use gas chromatography-mass spectrometry (GC-MS) by SPME.

The fifty-two aroma compounds detected by GC-O-MS in the sample from Hangbaizhi are listed in Table 1. The compounds represented a variety of smells, such as pine, camphor, woody, spice, herb, citrus, sour, and grass. Among these compounds, the content of terpenes, the main contributors to the fragrance of certain plants, was the highest. Terpenes may substantially contribute to the aroma, and thus the flavor, of Hangbaizhi. The content of aldehydes ( 9 derivatives) was second highest. The aromas of aldehydes can be described as fatty, cut-grass-like, and citrusy. Because aldehydes usually have low odor thresholds, they may contribute to the overall odor of A. dahurica. Terpenes (followed by aldehydes) were also the most abundant compounds extracted from Qibaizhi and Bobaizhi. Nineteen terpenes and 6 aldehydes were common aroma components among the three kinds of A.dahurica, which may cause the $A$. dahurica samples from different locations to have similar odor characteristics. 
Qibaizhi and Bobaizhi contained more kinds of alcohols than Hangbaizhi; however, due to their low contents or high thresholds, few alcohols were detected during the sniff tests. Aromatic species, ketones, acids, and esters detected in the A. dahurica samples may also contribute to their overall odor.

Table 1. Qualitative evaluation of the aroma compounds collected from A. dahurica using solid-phase microextraction (SPME), purge and trap (P\&T), stir bar sportive extraction (SBSE) and dynamic headspace sampling (DHS).

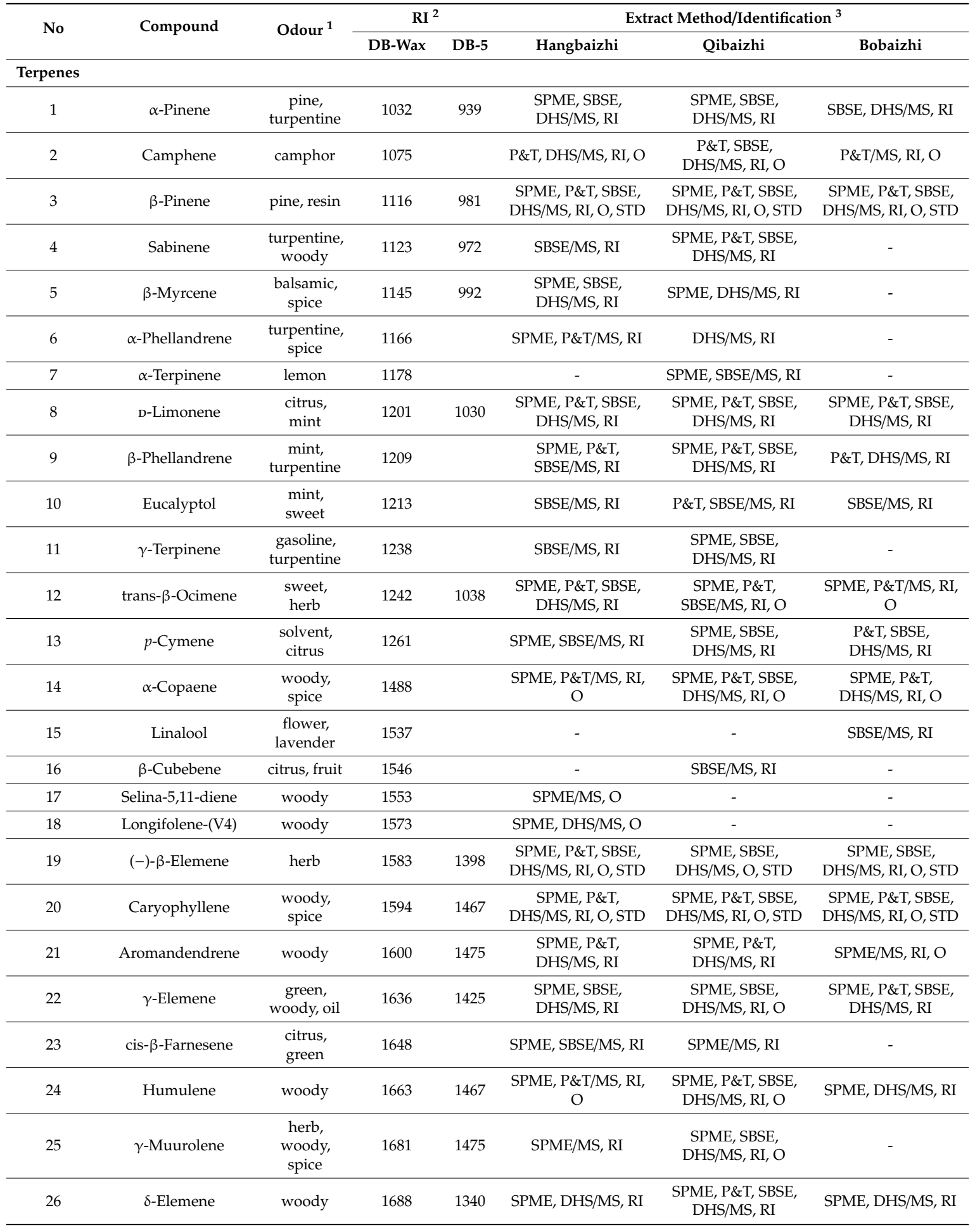


Table 1. Cont.

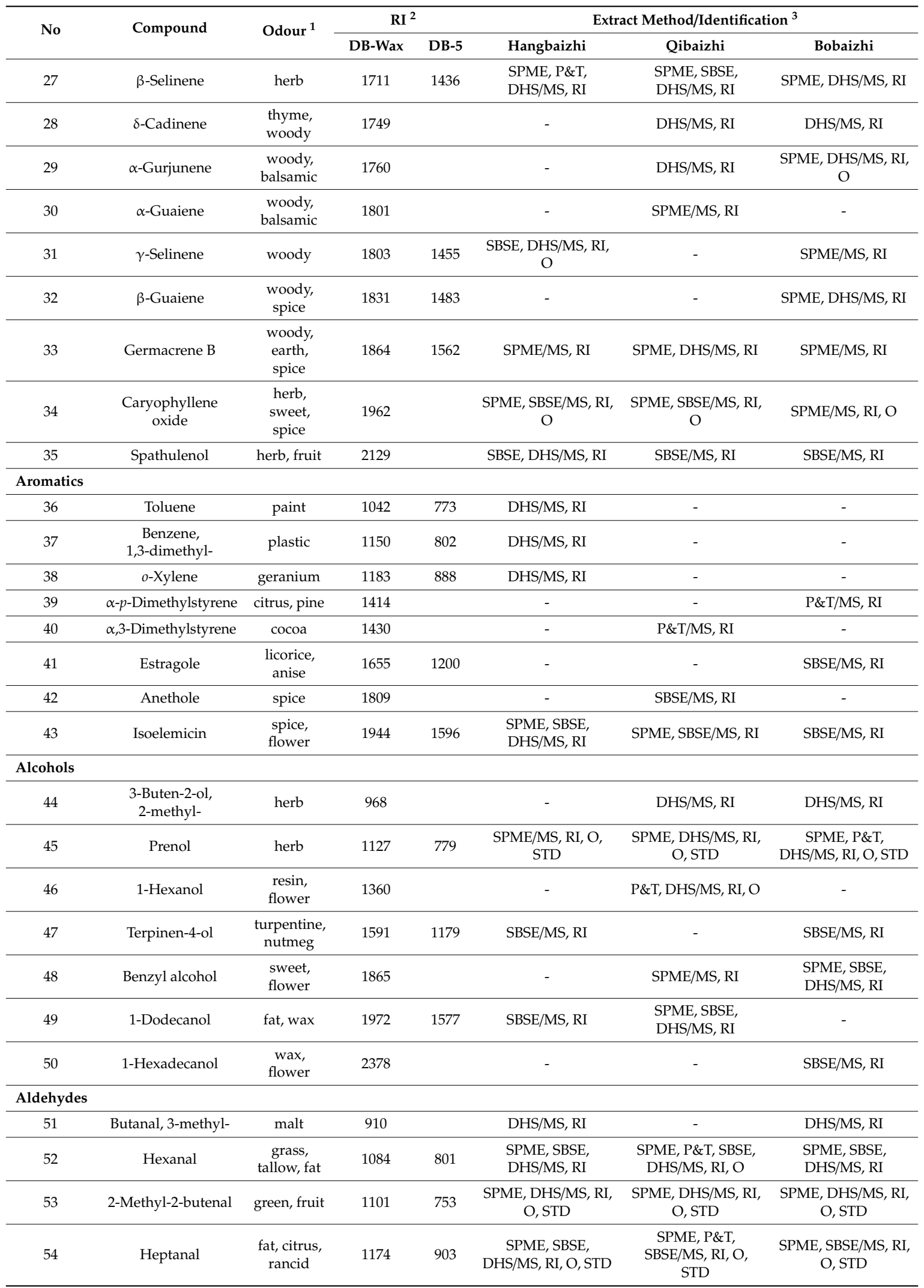


Table 1. Cont.

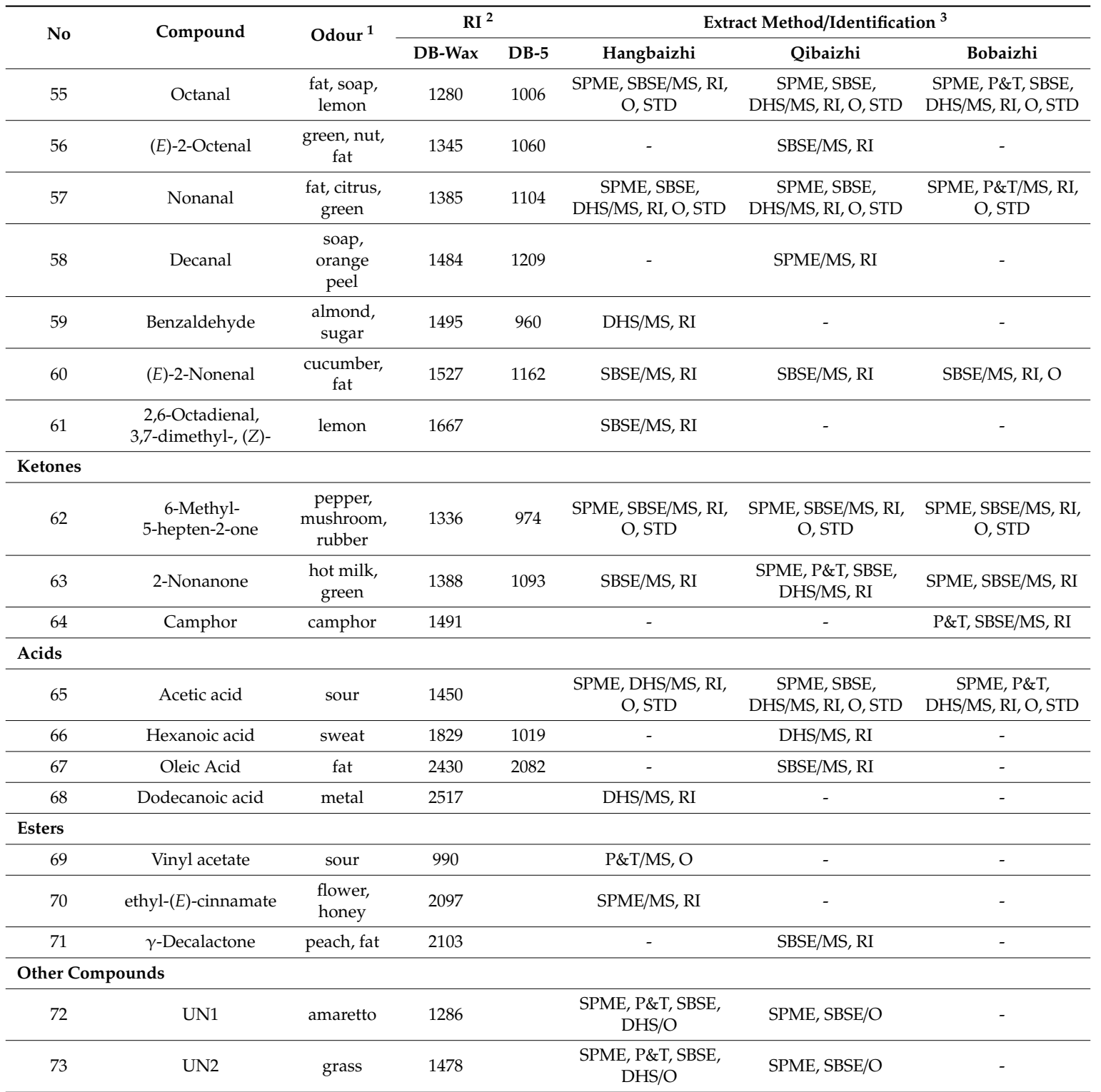

${ }^{1}$ Odor description was obtained by the combination of Flavornet website search and actual sniffing. ${ }^{2}$ Retention index (RI) for the odorant on DB-wax and DB- 5 columns. ${ }^{3}$ Methods of odorant identification included RI, MS, O, and STD, which represent the linear retention index, mass spectrum, odor properties and comparison to authentic standards by GC-O-MS. UN1, UN2 represent the compounds that could be sniffed by olfactometry but could not detected by mass spectrometry. 


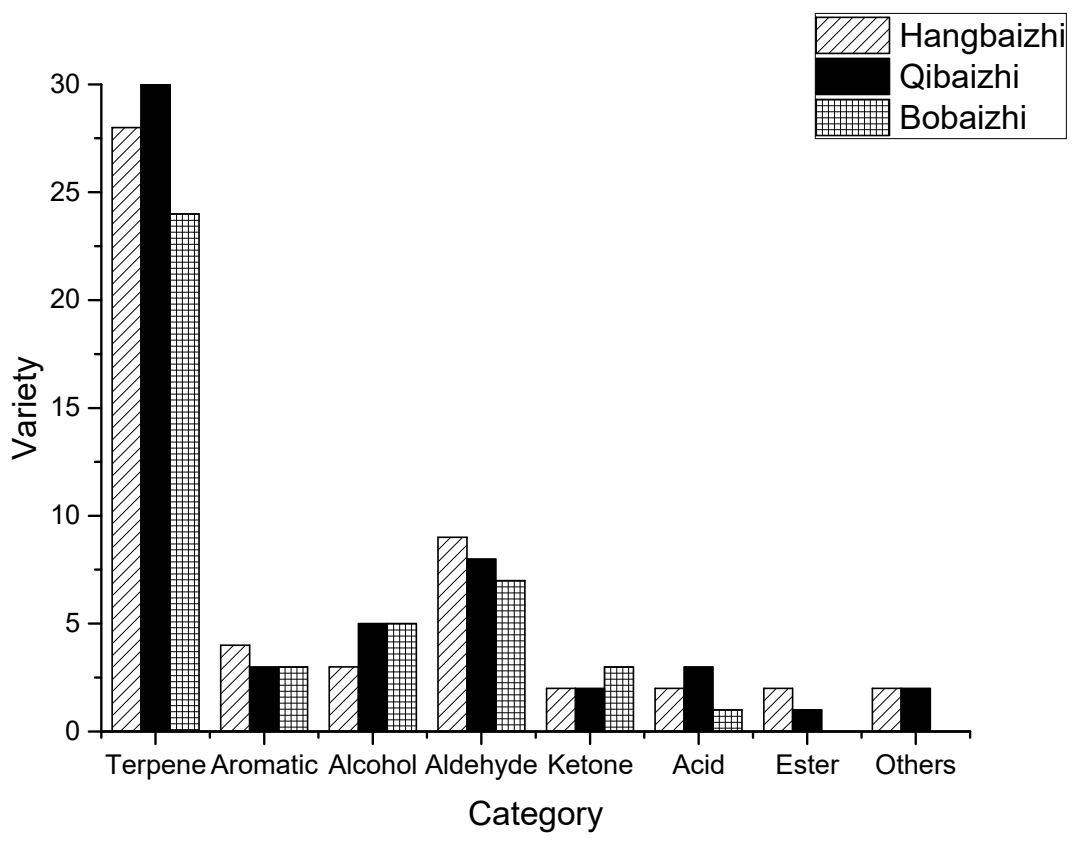

Figure 1. Types of flavor compounds extracted by SPME, P\&T, SBSE, and DHS from Hangbaizhi, Qibaizhi and Bobaizhi.

\subsection{Comparison of Quality of Aroma Compounds by the Different Extraction Methods}

As shown in Table 1, 31 common aroma compounds were identified in all three samples. A total of 34, 38, and 26 aroma compounds were extracted from Hangbaizhi, Qibaizhi, and Bobaizhi, respectively, by SPME; 15, 17, and 15 aroma compounds were extracted by P\&T; 29, 38, and 22 aroma compounds were extracted by SBSE, while 29,33 , and 23 aroma compounds were extracted by DHS. The variety of compounds extracted by P\&T was the lowest, whereas those extracted by the other methods were not significantly different.

As shown in Figure 2, the volatile compounds extracted by the four different headspace extraction methods were different. P\&T was unable to extract aromatics, alcohols, aldehydes, ketones, and acids from Hangbaizhi but extracted those compounds from Qibaizhi and Bobaizhi in low amounts. However, the amounts of these aroma compounds extracted from the three kinds of A. dahurica using SPME, SBSE, and DHS were all higher than those obtained using P\&T. This result indicated that although these aroma compounds were present in Hangbaizhi, P\&T may not be a suitable method for extracting the aroma-active compounds from A. dahurica. In addition, SPME, SBSE and DHS effectively extracted the terpenes and aldehydes. However, these three methods were not ideal for the extraction of aromatic species, alcohols, ketones, acids, and esters. Although there may have been low contents of these compounds in the samples, it is likely that the headspace extraction method may not effectively extract all types of aroma compounds; thus, incorporating a solvent extraction step into the method may be necessary. The SPME method is simple and consumes a small amount of material, while the SBSE and DHS methods require cold trapping technology, which not only consumes a larger amount of material but is also expensive. Thus, future research focusing on the extraction of aroma-active compounds from the roots of A. dahurica should be carried out using the SPME method. 


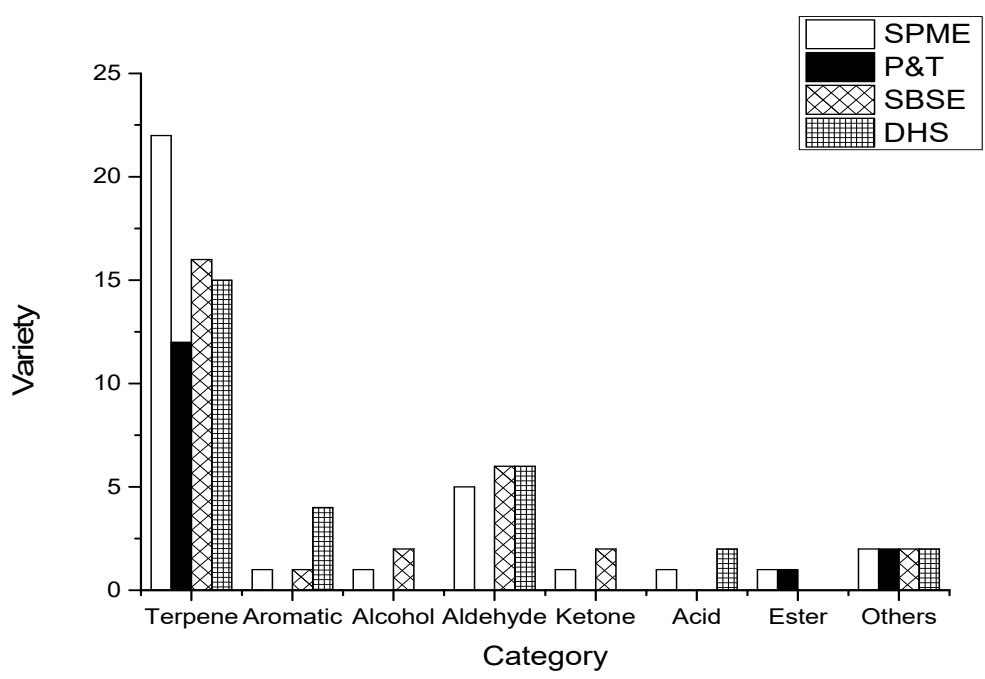

(A)

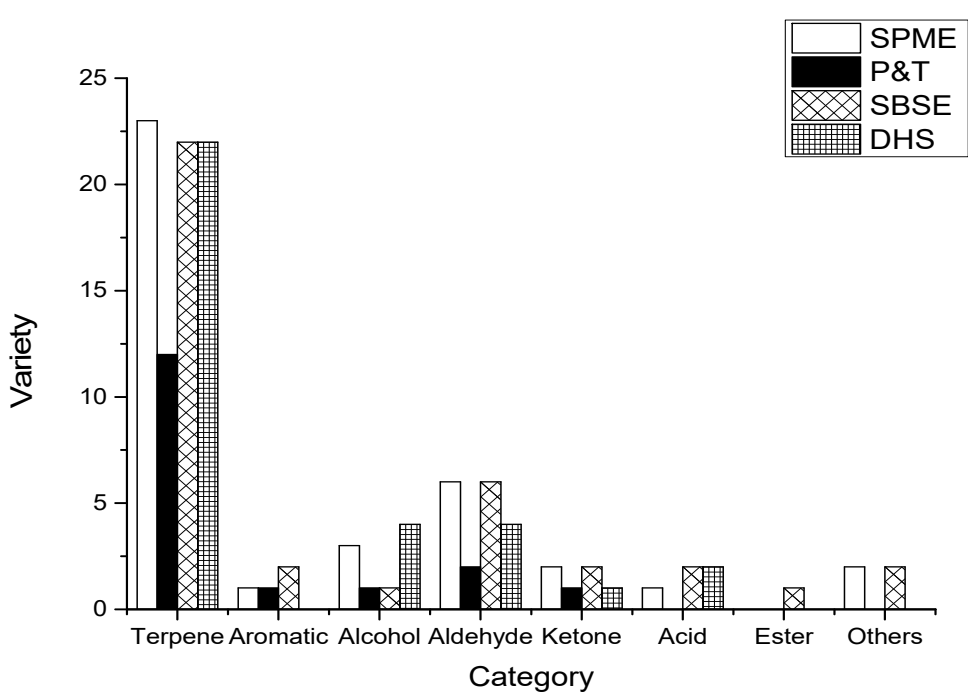

(B)

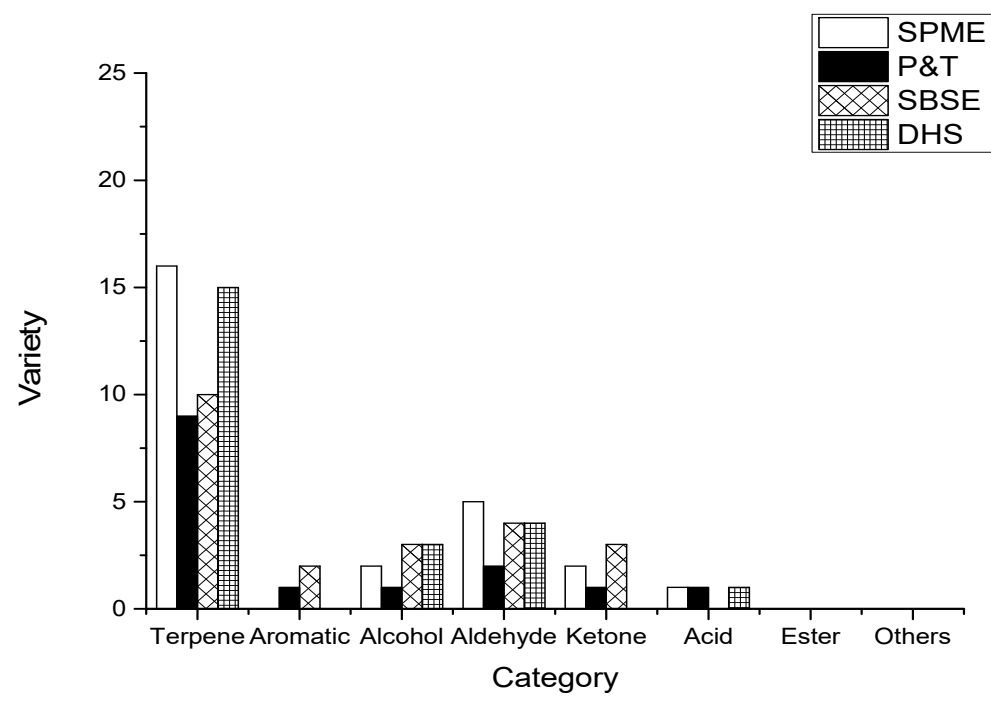

(C)

Figure 2. Quantities of the different kinds of flavor compounds extracted by SPME, P\&T, SBSE, and DHS from the three kinds of A. dahurica: (A) Hangbaizhi, (B) Qibaizhi, and (C) Bobaizhi. 


\subsection{Key Aroma-Active Compounds Identified by SPME-AEDA}

To further explore the key aroma-active compounds in the root of $A$. dahurica, dilution analysis was performed using SPME in conjunction with AEDA. As shown in Table 2, there were 12, 16, and 15 compounds that contributed to odors of in Hangbaizhi, Qibaizhi, and Bobaizhi, respectively, as determined by the sniffing test. The FD factor reflects the magnitude of the contribution of each aroma compound: A high FD factor corresponds to a greater contribution to the flavor of A. dahurica.

As shown in Table 2, the FD value of prenol (herbal) was higher in Hangbaizhi (FD = 81) than in the other samples. Nonanal (citrusy) and $\alpha$-copaene (woody) (FD = 27), as well as acetic acid (sour) and $\beta$-pinene (pine) $(F D=9)$, contributed substantially to the flavor of Hangbaizhi. Hence, these five compounds were key aroma-active compounds in Hangbaizhi. Sixteen aroma-active compounds were detected in Qibaizhi by the sniffing test. Prenol (herbal) (FD = 81), (-)- $\beta$-elemene (spicy) (FD = 81), $\beta$-pinene (pine) $(\mathrm{FD}=27), \gamma$-muurolene (herbal) $(\mathrm{FD}=27)$, acetic acid (sour) $(\mathrm{FD}=9)$, caryophyllene (woody) $(\mathrm{FD}=9)$, and humulene (woody) $(\mathrm{FD}=9)$ were key aroma-active compounds in Qibaizhi. Fifteen aroma compounds, including $\alpha$-gurjunene (woody) (FD = 81), prenol (herbal) $(\mathrm{FD}=81$ ), $\alpha$-copaene (woody) (FD = 27), nonanal (citrusy) (FD = 27), 2-methyl-2-butenal (fruity) (FD = 9), acetic acid (sour) $(\mathrm{FD}=9)$, aromadendrene (woody) $(\mathrm{FD}=9)$ and $\beta$-pinene (pine) $(\mathrm{FD}=9)$, were identified in Bobaizhi, indicating that they were key aroma-active compounds in Bobaizhi. Compounds with FD $\leq 3$ were considered to be minor contributors to the overall aroma, but these compounds did enhance the complexity of $A$. dahurica in some ways.

SPME-AEDA indicated that the key aroma-active compounds in the three types of $A$. dahurica were similar; however, some compounds had different FD factors. For example, the FD value of 2-methyl-2-butenal (fruity) $(\mathrm{FD}=9)$ in Bobaizhi was higher than those in Hangbaizhi $(\mathrm{FD}=1)$ and Qibaizhi (FD = 1). The FD values of $\beta$-pinene (pine) $(F D=27),(-)$ - $\beta$-elemene (spicy) $(F D=81)$, and caryophyllene (woody) $(\mathrm{FD}=9)$ in Qibaizhi were higher than those in Hangbaizhi $(\mathrm{FD}=9,1$, and 3 for $\beta$-pinene, (-)- $\beta$-elemene and caryophyllene, respectively) and Bobaizhi (FD $=9,1$, and 1 , for $\beta$-pinene, (-)- $\beta$-elemene and caryophyllene, respectively). The FD value of nonanal (citrusy) $(\mathrm{FD}=1)$ in Qibaizhi was lower than those in Hangbaizhi (FD = 27) and Bobaizhi (FD = 27). Among all, the intensity of the volatile compounds on the A. dahurica samples have not been studied yet.

As shown in Table 3, the external standard method was used to accurately quantify ten key aroma-active compounds shared by Hangbaizhi, Qibaizhi, and Bobaizhi as identified by SPME-AEDA. It can be seen from the table that the same aroma compounds had different FD values in the different A. dahurica cultivars due to the difference in their concentrations. These common aroma-active compounds provided the three kinds of $A$. dahurica with similar odor properties, but because of their different concentrations in each sample, they contributed to the overall odors to different extents. In addition to these ten common aroma compounds, the three kinds of $A$. dahurica each had several unique aroma-active compounds. For example, $\gamma$-muurolene (herbal) and humulene (woody) were key aroma-active compounds characteristic of Qibaizhi, and $\alpha$-gurjunene (woody) was an aroma-active compound unique to Bobaizhi. These differences may have caused differences in the overall odors of these three kinds of $A$. dahurica. 
Table 2. Dilution analysis of $A$. dahurica using SPME combined with AEDA.

\begin{tabular}{|c|c|c|c|c|c|c|c|c|}
\hline \multirow{2}{*}{ No } & \multirow{2}{*}{ Compound } & \multirow{2}{*}{ CAS } & \multicolumn{2}{|c|}{ RI } & \multirow{2}{*}{ Odor } & \multicolumn{3}{|c|}{ FD } \\
\hline & & & DB-Wax & DB-5 & & Hangbaizhi & Qibaizhi & Bobaizhi \\
\hline 1 & 2-Methyl-2-butenal & $1115-11-3$ & 1101 & 753 & green, fruity & 1 & 1 & 9 \\
\hline 2 & $\beta$-Pinene & $127-91-3$ & 1116 & 981 & $\begin{array}{l}\text { pine, resin, } \\
\text { turpentine }\end{array}$ & 9 & 27 & 9 \\
\hline 3 & Prenol & $556-82-1$ & 1127 & 779 & herb & 81 & 81 & 81 \\
\hline 4 & Heptanal & $111-71-7$ & 1174 & 903 & $\begin{array}{l}\text { fatty, citrusy, } \\
\text { rancid }\end{array}$ & 1 & 1 & 1 \\
\hline 5 & trans- $\beta$-Ocimene & $3779-61-1$ & 1242 & 1038 & sweetie, herb & - & 1 & 1 \\
\hline 6 & Octanal & $124-13-0$ & 1280 & 1006 & $\begin{array}{l}\text { fatty, soap, } \\
\text { lemon }\end{array}$ & 1 & 1 & 1 \\
\hline 7 & 6-Methyl-5-hepten-2-one & $110-93-0$ & 1336 & 974 & mushroom & 1 & 1 & 1 \\
\hline 8 & Nonanal & $124-19-6$ & 1385 & 1104 & citrusy, green & 27 & 1 & 27 \\
\hline 9 & Acetic acid & 64-19-7 & 1450 & & sour & 9 & 9 & 9 \\
\hline 10 & $\alpha$-Copaene & $3856-25-5$ & 1488 & & woody, spice & 27 & 3 & 27 \\
\hline 11 & Caryophyllene & $87-44-5$ & 1594 & 1467 & woody, spice & 3 & 9 & 1 \\
\hline 12 & $(-)$ - $\beta$-Elemene & 515-13-9 & 1595 & 1398 & spice & 1 & 81 & 1 \\
\hline 13 & Aromadendrene & $489-39-4$ & 1600 & 1475 & woody & - & - & 9 \\
\hline 14 & $\gamma$-Elemene & 29873-99-2 & 1636 & 1425 & $\begin{array}{c}\text { green, } \\
\text { woody, oil }\end{array}$ & - & 1 & - \\
\hline 15 & Humulene & $6753-98-6$ & 1663 & 1467 & woody & - & 9 & - \\
\hline 16 & $\gamma$-Muurolene & $30021-74-0$ & 1681 & 1475 & $\begin{array}{l}\text { herb, woody, } \\
\text { spice }\end{array}$ & - & 27 & - \\
\hline 17 & $\alpha$-Gurjunene & $489-40-7$ & 1760 & & woody & - & - & 81 \\
\hline 18 & Caryophyllene oxide & $1139-30-6$ & 1962 & & $\begin{array}{l}\text { herb, } \\
\text { sweetie, } \\
\text { spice }\end{array}$ & 3 & 3 & 1 \\
\hline
\end{tabular}

Table 3. Quantitative determination of the key aroma-active compounds collected from A. dahurica.

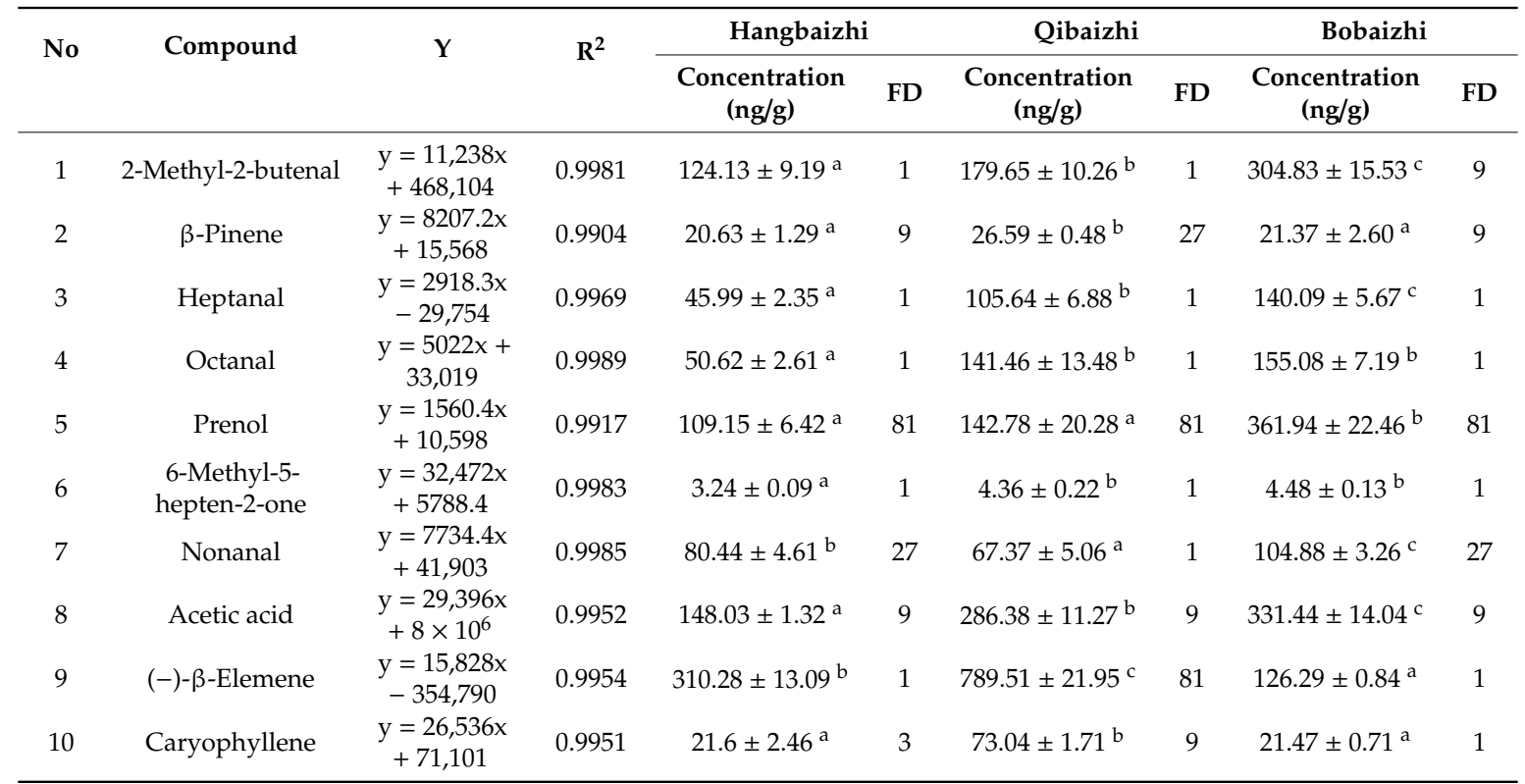

Values marked by the lower-case superscript letters (from " $\mathrm{a}$ " to " $\mathrm{c}$ ") within a line denote statistically significant differences $(p<0.05)$. 


\subsection{Sensory Evaluation}

The sensory evaluation data shown in Figure 3 indicate that the main characteristic aromas of the three types of $A$. dahurica were herbal, spicy and woody. All three types of A. dahurica had high scores for herbal aroma. As indicated by GC-O, the herbal aroma was due to prenol, caryophyllene oxide, and $\gamma$-muurolene. The spicy aroma in Qibaizhi was more intense than that in the other samples due to the presence of (-)- $\beta$-elemene, as indicated by GC-O. The woody aroma of Bobaizhi had a higher score than that of the other samples; $\alpha$-gurjunene, $\alpha$-copaene and aromadendrene contributed to the woody odor of Bobaizhi. The contents of the woody flavor compounds in Hangbaizhi and Qibaizhi were lower. Other low-scoring aromas, such as citrus, were generated by other active-aroma compounds. This conclusion was based on the presence of these aroma-active compounds and their various aromas, which were detected in the sniffing tests, and thus these compounds contributed to the complex aroma of $A$. dahurica.

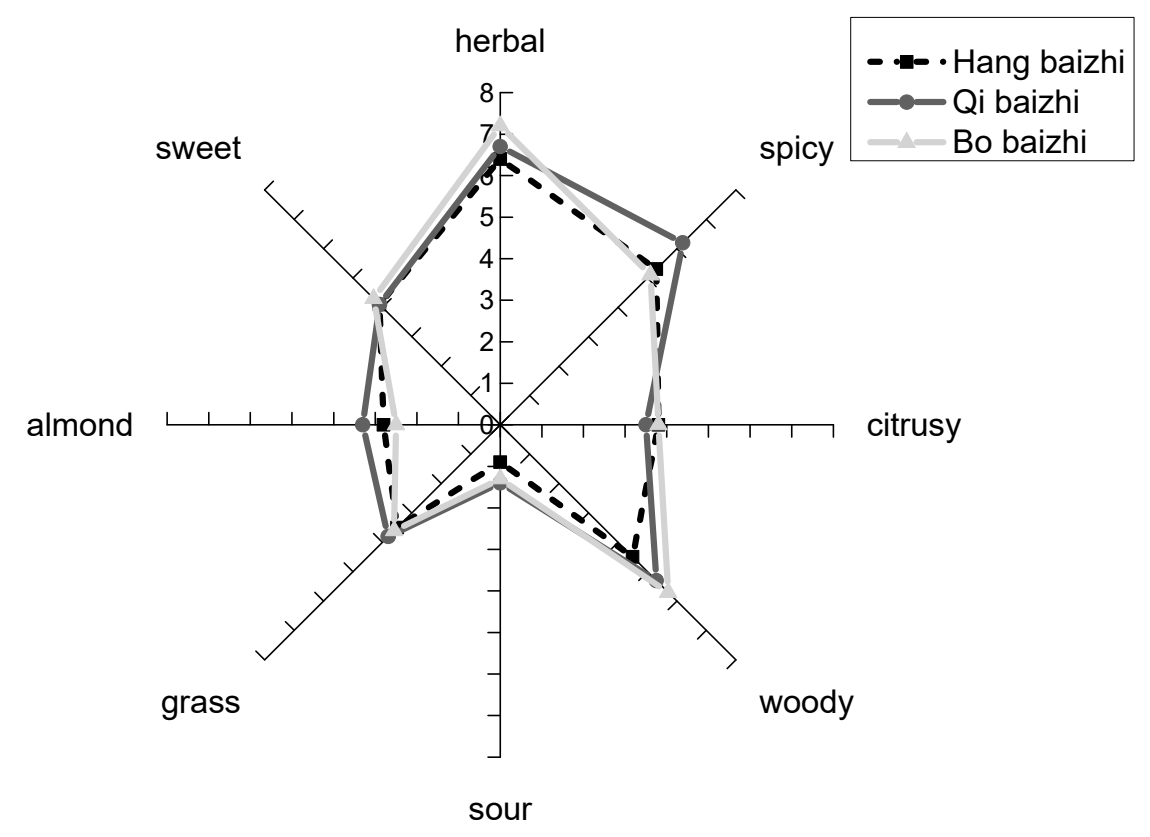

Figure 3. Odor sensory profiles of the three kinds of A. dahurica.

\subsection{PCA and PLS-DA of the Aroma Compounds of A. dahurica}

PCA and PLS-DA were performed to determine the differences in the key aroma compounds in the three kinds of $A$. dahurica. The concentrations of the ten shared key aroma compounds were used for PCA and PLS-DA. PCA score plots provide useful information about clustering samples into groups [16]. As shown in Table 4, three principal components were extracted, and these components represented $99.1 \%$ of the variation. The first, second and third main factors explained $57.7 \%, 39.7 \%$, and $1.72 \%$ of the total variance, respectively; PC1 and PC2 were sufficient to explain the maximum variation in all the original samples of $A$. dahurica with a combined contribution of $97.4 \%$. Figure 4 shows the PCA score plots of A. dahurica. The Hangbaizhi was located on the left while the Bobaizhi was located on the wright side, and the Qibaizhi was the upper middle portion of the correlation plot, indicating that they have the difference on aroma, three different kinds of $A$. dahurica can be clearly divided into three groups.

PLS-DA is a chemometric technique used to optimize the separation between different groups of samples; its loading plots reflect the relationships among important variables specific to the group of interest, and variable importance on projection (VIP) can be used to identify the most important variables $[17,18]$. The resulting loading plot (PC1vs PC2) is shown in Figure 5, for the investugation of relationships of the aroma compounds and classification of Baizhi, the aroma compounds $\beta$-pinene 
(No.2), (-)- $\beta$-elemene (No.9) and caryophyllene (No.10) were clustered with Qibaizhi (class 2); which were located at the same area, and it was indicated that these compounds were positive correlation with Qibaizhi. The seven aroma compounds such as the 2-methyl-2-butenal (No.1), prenol (No.5), etc., related to Bobaizhi, were in the lower right portion of the diagram.

Table 4. Eigenvalues of the four principal components, their contributions, and the cumulative contribution.

\begin{tabular}{cccc}
\hline Principal Component & Eigenvalue & Contribution (\%) & Cumulative Contribution (\%) \\
\hline 1 & 5.19 & 57.7 & 57.7 \\
2 & 3.57 & 39.7 & 97.4 \\
3 & 0.155 & 1.72 & 99.1 \\
\hline
\end{tabular}

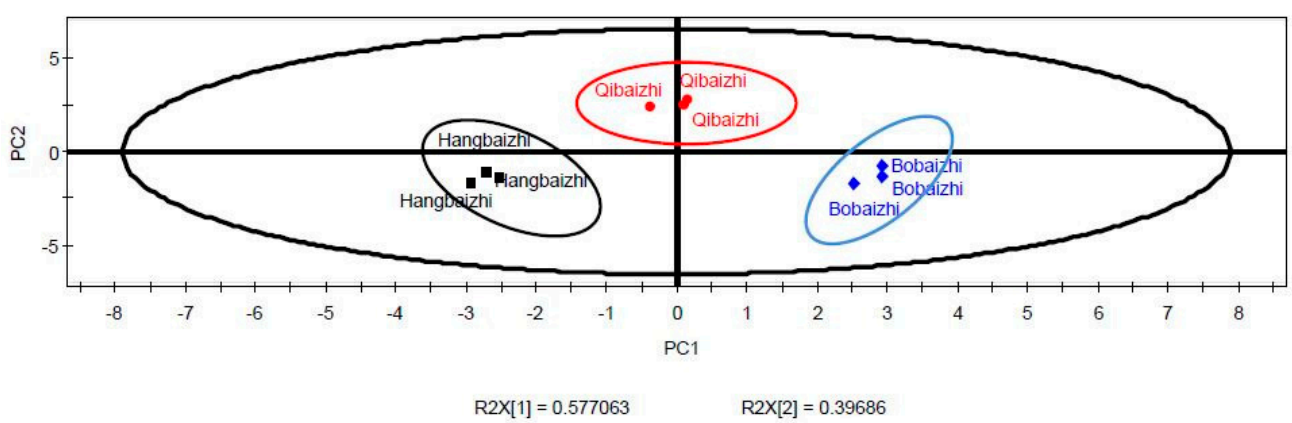

Figure 4. Scores plot of aroma-active compounds of $A$. dahurica by principal component analysis.

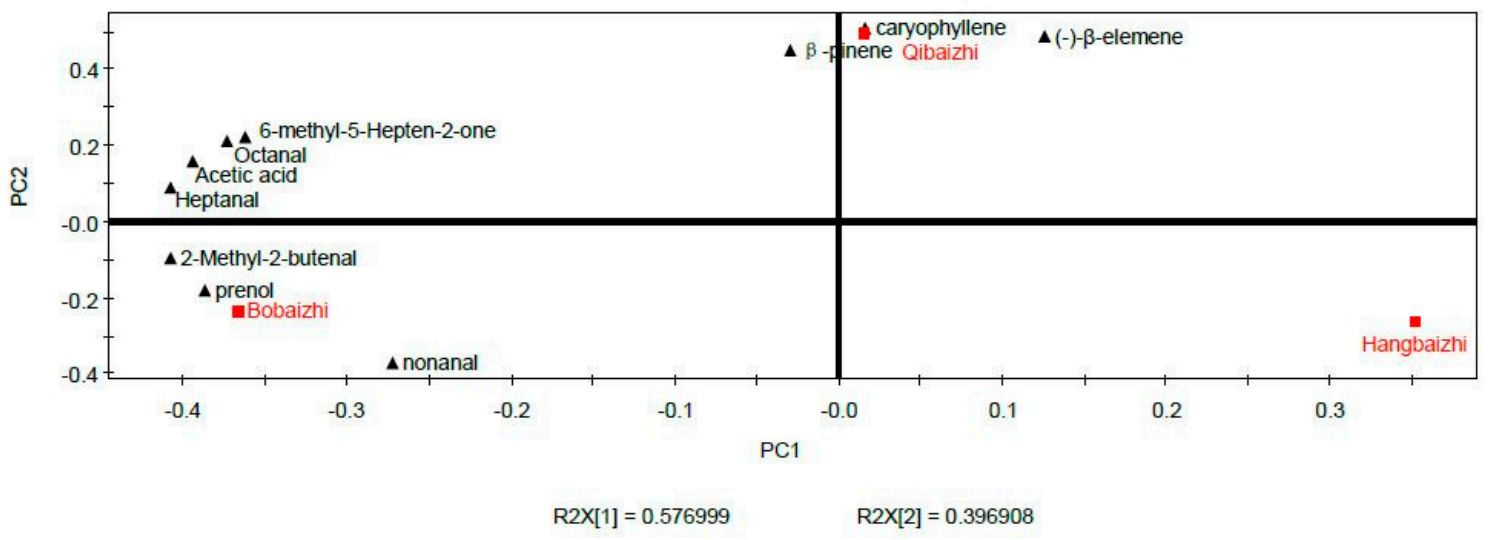

Figure 5. Loading plot of aroma-active compounds of A. dahurica by Partial least squares discriminant analysis.

\section{Materials and Methods}

\subsection{Preparation of $A$. dahurica}

Three types of dry A. dahurica root, including Hangbaizhi, Qibaizhi, and Bobaizhi root, were purchased from Beijing Tongrentang and were identified as genuine products by Professor Meng Xiansheng of the Liaoning University of Traditional Chinese Medicine (Dalian, China). Prior to analysis, each sample was ground into a powder using an electric grinder and then passed through a 40-mesh sieve. The samples were kept at room temperature. The sieved powder was transferred to a sealed bag and then stored in a dark dry place. 


\subsection{Chemicals}

$N$-Alkanes (C7-C30) used to calculate the retention indices (RI values) were obtained from Sigma-Aldrich (Milwaukee, WI, USA). All standards ( $\beta$-pinene, (-)- $\beta$-elemene, prenol, 2-methyl-2-butenal, heptanal, octanal, nonanal, 6-methyl-5-hepten-2-one, caryophyllene, and acetic acid) used for qualitative and quantitative analysis of the aroma compounds were purchased from Sigma-Aldrich. Nitrogen (99.9992\% purity) and helium (99.999\% purity) were purchased from Beijing Haipubeifen Gas Industry Co. Ltd. (Beijing, China). Liquid nitrogen (99.99\% purity) was purchased from Beijing Xianheyu Gas Industry Co. Ltd. (Beijing, China).

\subsection{Extraction of the Volatile Compounds}

Four different headspace extraction methods (SPME, P\&T, SBSE, and DHS) were employed to isolate the volatile compounds from $A$. dahurica. The extracted volatile compounds were then subjected to GC-O-MS analysis, and the results were compared.

\subsubsection{Extraction of the Aroma Compounds from A. dahurica by SPME}

SPME was carried out according to the method described by Lee, Cho, and Lee, K.G. [19] with minor modifications. Three grams of A. dahurica was weighed in a $40-\mathrm{mL}$ odor-free headspace vial. The volatile compounds were extracted at $60^{\circ} \mathrm{C}$ for $40 \mathrm{~min}$ with an equilibration time of $20 \mathrm{~min}$ by static SPME using 1-cm fibers coated with 50/30 $\mu \mathrm{m}$ divinylbenzene/carboxen on polydimethylsiloxane (DVB/CAR/PDMS) (57329-U; Supelco, Bellefonte, PA, USA). Then, the fibers were subjected to analysis by GC-O-MS at a temperature of $250{ }^{\circ} \mathrm{C}$ for $5 \mathrm{~min}$. Each experiment was conducted in triplicate.

\subsubsection{Extraction of the Aroma Compounds from A. dahurica by P\&T}

The extraction by P\&T was conducted following the method described in Cai and Huang [20] with minor modifications. Three grams of A. dahurica was weighed in a 40-mL odor-free headspace vial. The extraction conditions were as follows: purge temperature, $60^{\circ} \mathrm{C}$; equilibration time, $20 \mathrm{~min}$; purge time, $40 \mathrm{~min}$; flow rate of nitrogen, $50 \mathrm{~mL} / \mathrm{min}$; desorption temperature, $250{ }^{\circ} \mathrm{C}$; and desorption time, $2 \mathrm{~min}$. Then, the extracted volatile compounds were subjected to analysis by GC-O-MS. Each experiment was conducted in triplicate.

\subsubsection{Extraction of the Aroma Compounds from A. dahurica by SBSE}

SBSE was carried out according to the method described by Hoz, Salinas, and Ferrandino [15] with minor modifications. Three grams of $A$. dahurica, weighed into a $40-\mathrm{mL}$ odor-free headspace vial, was dissolved in $20 \mathrm{~mL}$ of ultrapure water. The extraction was conducted at $60^{\circ} \mathrm{C}$, and the sample was equilibrated for $20 \mathrm{~min}$. HS-SBSE was carried out by exposing a commercial polydimethylsiloxane (PDMS)-coated stir bar to the sample in a hermetically sealed headspace vial, and the sample was stirred at $500 \mathrm{rpm}$ and heated to $60^{\circ} \mathrm{C}$ for $40 \mathrm{~min}$. After that, the stir bar was rinsed with pure water and then dried with a lint-free tissue. The sample introduction system was composed of an automated thermal desorption unit (TDU) combined with a multipurpose sampler (MPS) and a cold injection system (CIS). The sample was analyzed using GC-O-MS. Each experiment was conducted in triplicate.

The heating program for the TDU was as follows: The initial temperature of $50^{\circ} \mathrm{C}$ was held for $1 \mathrm{~min}$, then it was increased to $200{ }^{\circ} \mathrm{C}$ at $60^{\circ} \mathrm{C} / \mathrm{min}$ and held for $10 \mathrm{~min}$. Finally, the temperature was increased to $300^{\circ} \mathrm{C}$ at $60^{\circ} \mathrm{C} / \mathrm{min}$ and held for $10 \mathrm{~min}$.

The heating program for CIS was as follows: The temperature of the CIS was decreased to $-100^{\circ} \mathrm{C}$ using liquid nitrogen and then held until analysis using the TDU was completed. After that, the temperature was increased to $240^{\circ} \mathrm{C}$ at $10^{\circ} \mathrm{C} / \mathrm{min}$ and held for $1 \mathrm{~min}$. 


\subsubsection{Extraction of the Aroma Compounds from A. dahurica by DHS}

The DHS analysis was performed following the method described by Waehrens and Zhang [21] with minor modifications. Ten grams of $A$. dahurica was weighed in a $150-\mathrm{mL}$ odor-free dynamic headspace bottle and placed in a circulating water bath at $60^{\circ} \mathrm{C}$ for $20 \mathrm{~min}$. After that, the sample was purged with high-purity nitrogen at a flow rate of $100 \mathrm{~mL} / \mathrm{min}$ and then dynamically absorbed onto a Tenax column for $40 \mathrm{~min}$. The volatile components adsorbed on the Tenax column were then injected through an MPS multisampler, where they were separated and analyzed by a thermal desorption system (TDU), a cold injection system (CIS), and GC-O-MS. Each experiment was conducted in triplicate.

The conditions for the TDU and CIS were consistent with those used in the SBSE method.

\subsubsection{GC-O-MS Analysis}

In this work, GC-MS/MS (7890A-7000B; Agilent Technologies Inc., Santa Clara, CA, USA) was employed to analyze the aroma compounds, and a Sniffer 9000 Olfactometer (Brechühler, Schlieren, Switzerland) was used to detect their aromas. A DB-Wax (strong polarity, $30 \mathrm{~m} \times 0.25 \mathrm{~mm} \times 0.25 \mu \mathrm{m}$; J\&W Scientific, Folsom, CA, USA) and DB-5 (weak polarity, $30 \mathrm{~m} \times 0.25 \mathrm{~mm} \times 0.25 \mu \mathrm{m}$; J\&W Scientific, Folsom, CA, USA) column were used to separate the volatile compounds. The method was conducted according to Song and Liu (2018) with minor modifications. Ultrahigh-purity helium was used as the carrier gas at a flow rate of $1.2 \mathrm{~mL} / \mathrm{min}$. The heating program was as follows: The initial temperature was set at $40^{\circ} \mathrm{C}$ and held for $3 \mathrm{~min}$, then it was increased to $200^{\circ} \mathrm{C}$ at a rate of $5{ }^{\circ} \mathrm{C} / \mathrm{min}$ and finally raised to $230{ }^{\circ} \mathrm{C}$ at a rate of $10{ }^{\circ} \mathrm{C} / \mathrm{min}$ and held for $3 \mathrm{~min}$. The split ratio was 5:1, and the injector and GC-MS/MS interface temperatures were set at 250 and $280^{\circ} \mathrm{C}$, respectively. Electron impact mass spectra were generated at $70 \mathrm{eV}$, and the $\mathrm{m} / \mathrm{z}$ scan was carried out from 50 to $350 \mathrm{~m} / \mathrm{z}$. The ion source temperature was set at $230^{\circ} \mathrm{C}$.

GC-O was carried out by five experienced assessors. The assessors smelled the aromas with sniffing masks and then recorded the aroma they perceived; the retention time, intensity value, and description of the aroma were all noted. Each assessor sniffed each sample twice. The compounds were considered to be aroma active when odors with the same retention time were detected by at least half of the assessors [22].

\subsection{Qualitative Analysis of the Volatile Compounds in the Root of A. dahurica}

The chemical identification was conducted by comparison to a database of mass spectra and the linear RI values reported in the literature, and the aromatic characteristics were established from references and sniffing tests. Some key aroma-active compounds were also revalidated by comparing their profiles to those of standard compounds. The RI values were calculated as follows based on a normal alkane series and comparison with references:

$$
\mathrm{RI}=100 n+100 n\left(\mathrm{t}_{\mathrm{a}}-\mathrm{t}_{n}\right) /\left(\mathrm{t}_{n+1}-\mathrm{t}_{n}\right)
$$

where ta represents the retention time of sample $a, t_{n}$ represents the retention time of the normal alkane series, and $n$ is the number of carbon atoms [23].

\subsection{SPME-AEDA}

SPME-AEDA is similar to the method described by Kim et al. [24], in which dilution analysis can be achieved by changing the split ratio in the GC-O method. In this study, the split ratios of the GC-O method were 5:1, 15:1, 45:1, 135:1, and 405:1, which corresponded to FD factors of 1, 3, 9, 27, and 81, respectively. The assessors sniffed the effluents from the separations with different split ratios through sniffing masks, and the process was stopped when an aroma could not be detected; the results are expressed as the FD factor. The FD factor is related to the intensity of the odor and thus can indicate the contribution of a compound to the overall smell. 


\subsection{Quantitative Analysis of the Key Aroma Compounds in the Root of A. dahurica}

In this study, the external standard method was used to accurately quantify the key aroma-active compounds shared by the three A. dahurica root samples analyzed by SPME-AEDA. The method used in this work was that described by Tominaga and Dubourdieu [25] with some modifications. The aroma compounds in the roots of $A$. dahurica were first extracted by SPME, and then, the key aroma compounds were analyzed by GC-O-MS in selective ion mode (SIM). The roots of A. dahurica were used as a matrix, and a mixed standard was added at different concentrations. Then, SPME was used for extraction, and GC-O-MS in SIM was used to analyze the key aroma compounds. The concentration of the compound to be quantified in the mixed standard was plotted along the $x$-axis, and the difference between the peak area of the matrix spiked with the mixed standard and that of the blank matrix was plotted along the $y$-axis. By adjusting the concentration of the external standards added to the sample, a standard curve was prepared. The compounds were accurately quantified using the standard curves. The standard curves of these compounds were calibrated by subtracting the blank ratios (the area of the peak of a selected ion of the compound found naturally in the root of A. dahurica).

\subsection{Sensory Evaluation}

Three grams of the A. dahurica sample was accurately weighed in a $40-\mathrm{mL}$ headspace bottle. The sensory evaluation was carried out in a quiet, tasteless sensory evaluation room by ten trained assessors ( 6 women and 4 men with an average age of 25 years old from the Laboratory of Molecular Sensory Science, Beijing Technology and Business University). The evaluators did not consume food for at least one hour prior to evaluation, and during the evaluation, they were not allowed to communicate with each other. The interval time between each sample was $5 \mathrm{~min}$. The intensity of each aroma was evaluated on a 10-point intensity scale: 1 indicated a weak intensity, 5 indicated a moderate intensity, and 10 indicated a strong intensity [26].

\subsection{Statistical Analysis}

PCA and PLS-DA were conducted using SIMCA-P+ 11.0 software to classify the three kinds of A. dahurica and identify the key aroma-active compounds that most influenced the classification.

Tables were constructed using Microsoft Word 2010. Figures were constructed using Origin software (version OriginPro 9.1, OriginLab Inc., USA). The analyses of the volatile compounds of each sample using SPME, P\&T, SBSE, and DHS were carried out in triplicate.

\section{Conclusions}

It could be seen from this study that using SPME methods, the 34, 38, and 26 aroma compounds were extracted from Hangbaizhi, Qibaizhi, and Bobaizhi, indicating that they were more efficient methods than the other three extraction methods. Following this, the key aroma compounds were identified by SPME-AEDA, which were 20, 21, and 17 aroma-active compounds from these three samples, respectively. The Prenol, nonanal, $\alpha$-copaene, acetic acid, and $\beta$-pinene were the key aroma compounds in Hangbaizhi; prenol, (-)- $\beta$-elemene, $\beta$-pinene, $\gamma$-muurolene, acetic acid, caryophyllene, and humulene were the key aroma compounds of Qibaizhi; and $\alpha$-gurjunene, prenol, $\alpha$-copaene, nonanal, 2-methyl-2-butenal, acetic acid, aromadendrene, and $\beta$-pinene were the key aroma compounds in Bobaizhi. Sensory evaluation combined with GC-O indicated that the aromas of Baizhi included herb, spice, and woody. PCA and PLS-DA also showed that the three different kinds of Baizhi can be divided into three groups on the basis of the differences in the concentrations of their key aroma-active compounds, and caryophyllene, (-)- $\beta$-elemene, nonanal, and $\beta$-pinene play important roles in the classification.

This study systematically explored the differences in the flavor compounds of A. dahurica root samples originating from three different locations, key aroma compounds of each A. dahurica root sample, and the flavor characteristics of the different $A$. dahurica samples, providing a theoretical basis 
for its use as a spice. In future, studies are needed to investigate the reason that leads to the different flavor characteristics of different samples and to analyze the pathways of the aroma compounds.

Author Contributions: D.H. designed the experiment and analyzed the data. D.H. and T.L. wrote the paper. J.G., M.Z., and A.A. performed the experiments. T.Z., H.S., and A.A. commented the manuscript. All authors have read and approved the manuscript.

Funding: This research was funded by the Talent Training Quality Construction-First-Class Specialty Construction (municipal level)_Food Science and Engineering, grant number PXM2019_014213_000010. This research was funded by the Talent Training Quality Construction-First-Class Specialty Construction (municipal level)—Food Science and Engineering, grant number PXM2018_014213_000041.

Conflicts of Interest: The authors declare no conflict of interest.

\section{References}

1. Chau, C.F.; Wu, S.H. The development of regulations of Chinese herbal medicines for both medicinal and food uses. Trends Food Sci. Technol. 2006, 17, 313-323. [CrossRef]

2. Lu, M.; Yuan, B.; Zeng, M.; Chen, J. Antioxidant capacity and major phenolic compounds of spices commonly consumed in China. Food Res. Int. 2011, 44, 530-536. [CrossRef]

3. Wang, G.-H.; Chen, C.-Y.; Tsai, T.-H.; Chen, C.-K.; Cheng, C.-Y.; Huang, Y.-H.; Hsieh, M.-C.; Chung, Y.-C. Evaluation of tyrosinase inhibitory and antioxidant activities of Angelica dahurica root extracts for four different probiotic bacteria fermentations. J. Biosci. Bioeng. 2017, 123, 679-684. [CrossRef] [PubMed]

4. Pfeifer, I.; Murauer, A.; Ganzera, M. Determination of coumarins in the roots of Angelica dahurica by supercritical fluid chromatography. J. Pharm. Biomed. Anal. 2016, 129, 246-251. [CrossRef]

5. Wang, X.-H.; Xie, P.-S.; Lam, C.W.K.; Yan, Y.-Z.; Yu, Q.-X. Study of the destructive effect to inherent quality of Angelicae dahuricae radix (Baizhi) by sulfur-fumigated process using chromatographic fingerprinting analysis. J. Pharm. Biomed. Anal. 2009, 49, 1221-1225. [CrossRef]

6. Chinese Pharmacopoeia Commission. Pharmacopoeia of the People's Republic of China; Medical-Pharmaceutical Science \& Technology Publishing House: Beijing, China, 2010; p. 97.

7. Wei, L.; Zhan-Guo, L.; Dan, F.; Peng-Jun, W. Study on aroma components of baizhi angelica by HS-SPME and GC-MS. China Condiment. 2012, 37, 109-112.

8. Zhao, A.H.; Yang, X.B.; Yang, X.W.; Tao, H.Y.; Yu, J.L.; Wang, W.Q. GC-MS analysis of the chemical components of volatile oil from the root of Angelica dahurica. Chin. J. Pharm. Anal. 2012, 32, 763-768.

9. Li, L.; Lv, L.; Zhang, W.; Zhao, J.X.; Lv, D.Y.; Zhao, J.Y. GC-MS combined with PCA analysis of the essential oil from two varieties of Radix Angelicae Dahuricae. Chin. J. Pharm. Anal. 2011, 31, 112-118.

10. Matsushita, T.; Zhao, J.J.; Igura, N.; Shimoda, M. Authentication of commercial spices based on the similarities between gas chromatographic fingerprints. J. Sci. Food Agric. 2018, 98, 2989-3000. [CrossRef]

11. Sonmezdag, A.S.; Amanpour, A.; Kelebek, H.; Selli, S. The most aroma-active compounds in shade-dried aerial parts of basil obtained from Iran and Turkey. Ind. Crop. Prod. 2018, 124, 692-698. [CrossRef]

12. Omar, J.; Olivares, M.; Alonso, I.; Vallejo, A.; Aizpurua-Olaizola, O.; Etxebarria, N. Quantitative Analysis of Bioactive Compounds from Aromatic Plants by Means of Dynamic Headspace Extraction and Multiple Headspace Extraction-Gas Chromatography-Mass Spectrometry. J. Food Sci. 2016, 81, C867-C873. [CrossRef] [PubMed]

13. Raffo, A.; Masci, M.; Moneta, E.; Nicoli, S.; del Pulgar, J.S.; Paoletti, F. Characterization of volatiles and identification of odor-active compounds of rocket leaves. Food Chem. 2018, 240, 1161-1170. [CrossRef] [PubMed]

14. Stephan, A.; Bucking, M.; Steinhart, H. Novel analytical tools for food flavours. Food Res. Int. 2000, 33, 199-209. [CrossRef]

15. Hoz, K.S.D.L.; Salinas, M.R.; Ferrandino, A. Different coatings for the HS-SBSE grape volatile analysis in model solution: Preliminary results. Food Chem. 2016, 212, 814-820.

16. Zhao, Y.; Xie, Z.; Niu, Y.; Shi, H.; Chen, P.; Yu, L. Chemical compositions, HPLC/MS fingerprinting profiles and radical scavenging properties of commercial Gynostemma pentaphyllum (Thunb.) Makino samples. Food Chem. 2012, 134, 180-188. [CrossRef]

17. Barker, M.; Rayens, W. Partial least squares for discrimination. J. Chemom. 2003, 17, 166-173. [CrossRef] 
18. Gromski, P.S.; Muhamadali, H.; Ellis, D.I.; Xu, Y.; Correa, E.; Turner, M.L.; Goodacre, R. A tutorial review: Metabolomics and partial least squares-discriminant analysis-A marriage of convenience or a shotgun wedding. Anal. Chim. Acta 2015, 879, 10-23. [CrossRef]

19. Seonmi, L.; Heera, C.; Kwang-Geun, L. Volatile compounds as markers of tofu (soybean curd) freshness during storage. J. Agric. Food Chem. 2014, 62, 772-779.

20. Cai, M.G.; Huang, P.A. Purge and Trap Gas Chromatographic Method for Detection of Chlorofluorocarbons in Seawater. Chin. J. Anal. Chem. 2013, 41, 268-272. [CrossRef]

21. Waehrens, S.S.; Zhang, S.; Hedelund, P.I.; Petersen, M.A.; Byrne, D.V. Application of the fast sensory method 'Rate-All-That-Apply' in chocolate Quality Control compared with DHS-GC-MS. Int. J. Food Sci. Technol. 2016, 51, 1877-1887. [CrossRef]

22. Huanlu, S.; Jianbin, L. GC-O-MS technique and its applications in food flavor analysis. Food Res. Int. 2018, 114, 187-198.

23. Ye, L.; Hang, S.; Huan-Lu, S. Comparison of four extraction methods, SPME, DHS, SAFE, Versus SDE, for the analysis of flavor compounds in Natto. Food Anal. Methods 2018, 11, 343-354.

24. Kim, T.H.; Lee, S.M.; Kim, Y.S.; Kim, K.H.; Oh, S.; Lee, H.J. Aroma dilution method using GC injector split ratio for volatile compounds extracted by headspace solid phase microextraction. Food Chem. 2003, 83, 151-158.

25. Tominaga, T.; Dubourdieu, D. A novel method for quantification of 2-methyl-3-furanthiol and 2-furanmethanethiol in wines made from Vitis vinifera grape varieties. J. Agric. Food Chem. 2006, 54, 29-33. [CrossRef]

26. Zhu, M.; Li, E.; He, H. Determination of volatile chemical constitutes in tea by simultaneous distillation extraction, vacuum hydrodistillation and thermal desorption. Chromatographia 2008, 68, 603-610. [CrossRef]

Sample Availability: Not available.

(C) 2019 by the authors. Licensee MDPI, Basel, Switzerland. This article is an open access article distributed under the terms and conditions of the Creative Commons Attribution (CC BY) license (http://creativecommons.org/licenses/by/4.0/). 